\title{
Genomic and molecular alterations associated with early-onset and adolescent and young adult colorectal cancer
}

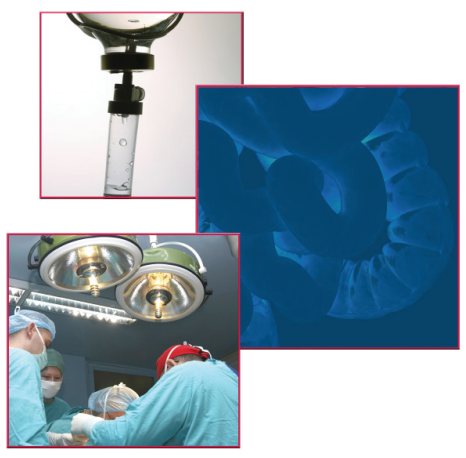

\author{
James V Tricoli*,1 \\ ${ }^{1}$ Cancer Diagnosis Program, Division of Cancer Treatment \& Diagnosis, National Cancer Institute, 6909 Medical Center Drive, \\ Rockville, MD 20892, USA \\ *Author for correspondence: tricolij@mail.nih.gov
}

While the incidence of colorectal cancer (CRC) in the US has declined at a pace of $3 \%$ annually between 2003 and 2012, there has been an increase in the incidence of early-onset colorectal cancer (EOCRC). The reasons for this increase are unclear. Diet, the environment, the microbiome and alcohol consumption have all been proposed as contributing factors. There is the possibility that EOCRC has a unique biology. Overlapping with the EOCRC age range is CRC in adolescent and young adults (AYA) that share many molecular characteristics with EOCRC. The purpose of this review is to cover current progress in our understanding of the biology of CRC in the context of adolescent and young adult CRC and EOCRC and discuss future directions.

First draft submitted: 6 April 2020; Accepted for publication: 11 September 2020; Published online: 21 October 2020

Keywords: AYACRC • chromosomal alterations • DNA repair pathways • EOCRC • LOCRC • miRNA • mutational variants

In general, the incidence rate of colorectal cancer (CRC) in the US has declined at a pace of $3 \%$ annually between 2003 and 2012 [1,2]. By contrast, there has been a documented increase between 1974 and 2015 in the incidence of early-onset colorectal cancer (EOCRC) in the US and Europe $[1,3,4]$. Interestingly, the rise is most prominent among Caucasians, particularly in the western US [2]. The exact reasons for this increase are currently unknown, although, factors such as higher rates of obesity, cigarette smoking, increased consumption of processed meat and alcohol consumption have all been implicated as possible causes [2]. Overlapping with the EOCRC age range of less than or equal to 50 years is the adolescent and young adult (AYA) age group, which encompass the 15-39 year age range [5,6]. Evidence suggests that CRC in the AYA age group may have a different molecular etiology than CRC diagnosed in older adults and may be more refractory to some treatment regimens than their older counterparts [7,8]. Like AYACRC, EOCRC tends to be associated with a higher percentage of synchronous and metachronous tumors, more poorly differentiated histology and mucinous and signet ring histology than that found in older adult patients [9,10]. However, the biological basis and clinical ramifications of these observations for both AYACRC and EOCRC are still incompletely defined. It is possible that some of the molecular factors associated with the unique characteristics of AYACRC are also involved in the spectrum of EOCRC disease. Such factors may be more prevalent in these cancers, or even unique to the disease.

Efforts to identify the underlying causes of EOCRC are increasing and include the identification of somatic mutational alterations unique to EOCRC, the impact of the microbiome on disease susceptibility and progression, alterations in genomic methylation patterns and the involvement of DNA repair pathway alterations - other than those associated with Lynch Syndrome. Differences in the number of chromosomal aberrations and breakpoints have also been observed between EOCRC and CRC in older adults [11]. The two recognized subtypes of EOCRC are the inherited subtype, characterized by Lynch Syndrome associated with micro satellite instability high (MSI$\mathrm{H}$ ), familial adenomatous polyposis (FAP) and $M U T Y H$ associated polyposis and the sporadic subtype, characterized by a lack of family history and the accumulation somatic mutations. The purpose of this review paper is to focus on the latter sporadic subtype and to review current progress in the understanding of the biology of CRC in the context of both EOCRC and AYACRC. We here focus on chromosomal alterations, somatic mutational variants 
and their affected signaling/DNA repair pathways, miRNA alterations and possible biomarkers and therapeutic targets as molecular factors associated with EOCRC and AYACRC.

\section{Chromosomal alterations \& gene expression}

Alteration of chromosome number and integrity is a hallmark of cancer and has been well-characterized in many forms of this disease. Methylation events have also been demonstrated to play a role in CRC, including the hypomethylation of long interspersed nuclear element-1 that can lead to the activation of proto-oncogenes [12]. Alterations in genomic methylation patterns, particularly hypomethylation events, are associated with increased chromosomal instability that contribute to tumorigenesis $[13,14]$. Numerous studies have examined the difference in variations at the chromosomal level in EOCRC and the concomitant changes in gene expression associated with those changes $[11,15]$. While there appears to be little difference in the fraction of the genome with chromosomal copy number alterations between EOCRC and late-onset CRC (LOCRC), one study that plotted gains and losses demonstrated that $3.2 \%$ of the genome is different between these two groups [11]. For example, loss occurred at the locus encoding the PRKAB2 gene (1q21.1), a regulatory unit of AMP-activated protein kinase, in $43 \%$ of EOCRC cases but in only $6 \%$ of LOCRC. Gains were also observed in EOCRC at chromosome 10q and 19q compared with LOCRC patients. In all, 16 genomic loci encoding $>500$ genes were observed to be preferentially altered in EOCRC compared with LOCRC [11]. This study also determined that $37 \%$ of genes had corresponding DNA copy number and RNA expression level alterations, and that among these genes, 107 mapped to genomic regions that differed in frequency of copy number change between EOCRC and LOCRC. Three genes $(C L C$, LTBP4, ZNF574) that map to a chromosome band (19q13.2) that showed copy number gain in EOCRC, also exhibited upregulation of mRNA expression. One of these $(C L C)$ encodes a phospholipase believed to be involved in inflammation [16]. This study also revealed five chromosomes that exhibited 15 loci that were more frequently altered in EOCRC compared with LOCRC, including MIR641 at 19q13.2, a miRNA that is known to be involved in CRC. Other studies have examined susceptibility loci in 191 cases of EOCRC compared with 1264 cases of LOCRC and determined that variants at 10p14 (rs10795668), 11q23.1 (rs3802842) and 15q13.3 (rs4779584) showed a different distribution between the two groups $[17,18]$. Additional studies have detected risk alleles for CRC associated with age at 8q24.21 (rs6983267), 10p14 (rs10795668) and 19q13.1 (rs10411210) [19] and at 11q23.1 (rs3802842), 14q22.2 (rs4444235) and 15q13.3 (rs4779584), 8q24.21 (rs6983267) and 20p12.3 (rs961253) [20]. A recent study compared the polygenic risk score, based on single-nucleotide variants (SNVs) associated with CRC in a cohort of 12,197 participants less than 50 years old and 95,865 participants 50 years of age or older [21]. That study determined the cumulative burden of SNVs associated with CRC strongly associated with EOCRC, particularly in the absence of a family history and, successfully identified participants at increased risk for EOCRC [21].

These and other studies demonstrate that there are numerous chromosomal alterations, and associated gene expression changes that are different in EOCRC when compared with LOCRC, which provides a foundation upon which to further build our understanding of the unique features of the early-onset form of this disease.

\section{Altered mutational frequencies associated with EOCRC}

The clinical presentation, histology, prognosis and ultimate outcome of EOCRC appears to differ from that observed in older adults. While delayed diagnosis may contribute to poor prognosis and outcome in this group, other factors may be involved. This is highlighted in Figure 1 where AYACRC, exclusive of hereditary predisposition syndromes, such as hereditary nonpolyposis colorectal cancer and FAP, is linked to fewer mutations and DNA repair deficits, as opposed to adult CRC where there is a serial accumulation of mutations [22-25]. The question is, does the biology of EOCRC vary from that found in older adults and if so how? The presence of single nucleotide variants SNVs, insertion/deletions (indels) and copy number variations are common across the spectrum of human cancers and consensus gene sets have been identified that exhibit consistent alterations in CRC [26,27]. The current data available from studies indicating a possible unique biology in EOCRC are limited [28-31]. Recent studies have pointed to a subset of gene variants that may be associated with EOCRC. These include increased variant frequencies observed for TP53, CTNNB1, POLE and a decreased variant frequency for APC, KRAS, BRAF, PIK3CA and FAM123B [28,29]. One study reported that in one cohort of patients ATM, TP53, FBXW7 and SMAD4 did not exhibit an age-related difference in mutation frequency, although only partial sequencing of the $A T M$ was conducted in that study, while in another cohort ATM mutations were more frequent in younger patients [31]. Another study has demonstrated that a combination of rare variants of the cyclin D1 gene, CCND1, contribute to an increased risk of multiple intestinal adenomas and of EOCRC [32]. A study has also implicated CTNNB1 as more frequently overexpressed 


\section{Concept of biologically different colon cancer} in older adolescents and young adults

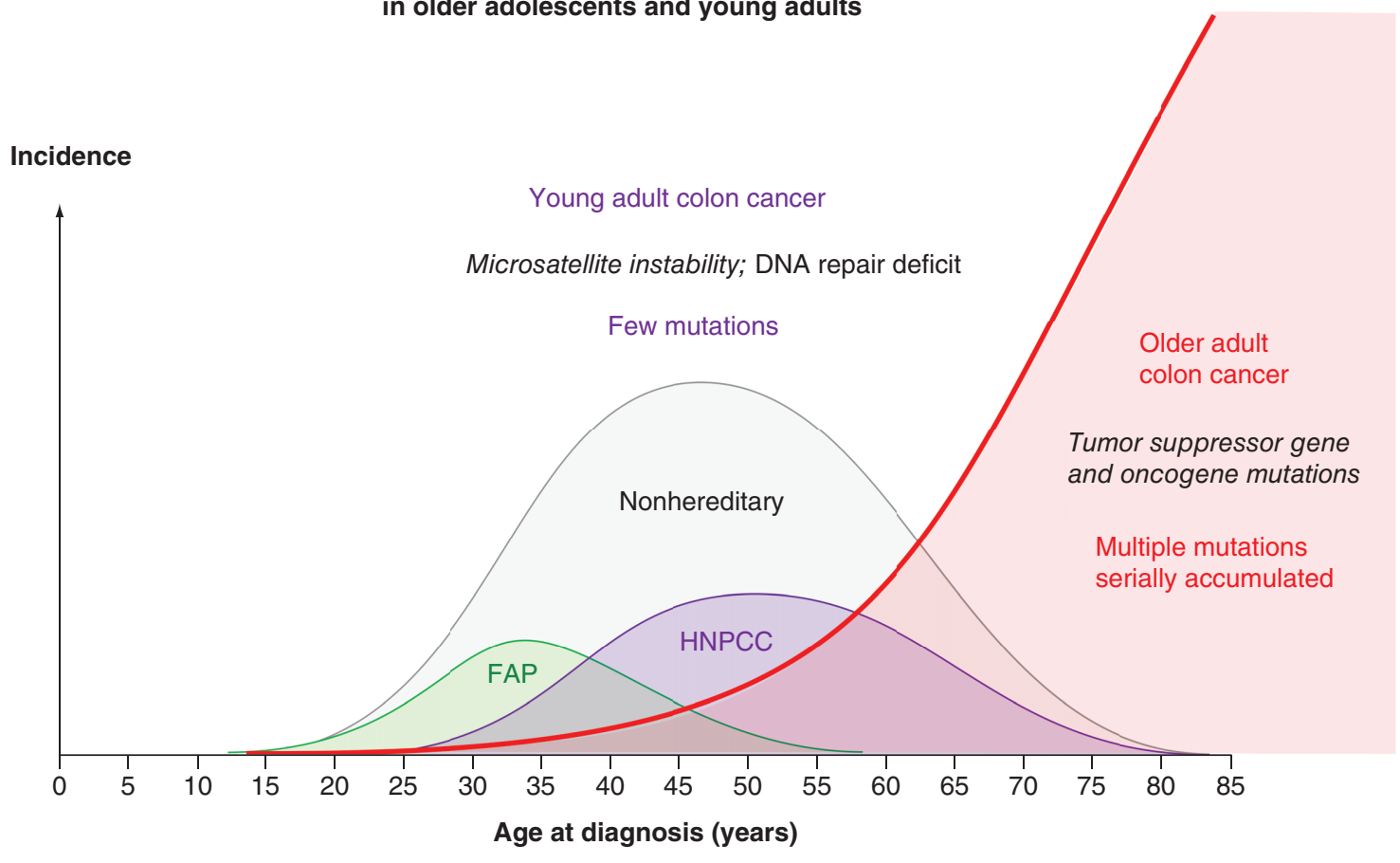

Figure 1. Distribution of incidence in nonhereditary colorectal cancer in the adolescent and young adult age group compared with colorectal cancer found in older adults. Data in-part derived from Jung et al. [22], Dalpatadu et al. [23], Jiang et al. [24] and Arriba et al. [25].

FAP: Familial adenomatous polyposis; HNPCC: Hereditary nonpolyposis colorectal cancer.

in EOCRC compared with LOCRC and the TNFR1 pathway as a key factor associated with EOCRC [33] in agreement with a previous study implicating immune response and inflammation in this form of the disease [34].

Some of the earliest evidence for the possibility of unique biology in AYA cancer came from studies of CRC in the AYA population, the age range of which overlaps significantly with that of EOCRC. Many AYACRC patients have a poorer prognosis, exhibit a more aggressive disease phenotype and have poorer survival than CRC in older adults [7]. In Figure 2 surveillance, epidemiology and end results (National Cancer Institute, MD, USA) data is shown, which demonstrates that survival is lower for colonic, rectal and anal carcinomas in AYAs (indicated by yellow highlighting) than when compared with older patients and, for each, inversely proportional to age within the AYA age range [35]. Like EOCRC, AYACRC exhibits a greater frequency of mucinous histology, signet ring cells, MSI-H and a higher incidence of mutations in mismatch-repair (MMR) genes [9,36-38]. A study by Hong et al. detected expression changes in genes from tumor adjacent normal mucosa in EOCRC patients compared with mucosa from healthy controls [39]. In that study FOS, FOSB, EGR1, VIP, CYR61, KRT24 and UCHL1 all displayed increased expression in the normal mucosa from EOCRC patients compared with controls. The functions of the proteins encoded by these genes include transcriptional regulation, angiogenesis, cell signaling and protein catabolism.

Relatively few molecular genetic studies in CRC have been conducted in the AYACRC population, perhaps because these cases are few-in-number and due to the paucity of tissues available for analysis. In addition, it should be noted that the majority of the next generation sequencing (NGS) studies that have been performed in AYACRC utilized targeted gene panels to discern these differences, rather than whole exome sequencing. The two most comprehensive analyses of gene variants in the AYACRC cohort suggest a variety of genes may be associated with the biology of AYACRC and some overlap with studies in EOCRC [40,41]. These include POLE and ATM, both of which are involved in DNA repair pathways [37]. In addition, $P O L E$ was identified as having a greater frequency of mutation in AYACRC compared with adult CRC by two independent studies utilizing different NGS platforms and different algorithmic analysis pipelines [40,41]. While one study [30] determined there was no difference in the frequency of FBXW7 mutations between EOCRC and LOCRC, two studies in AYACRC patients detected an 


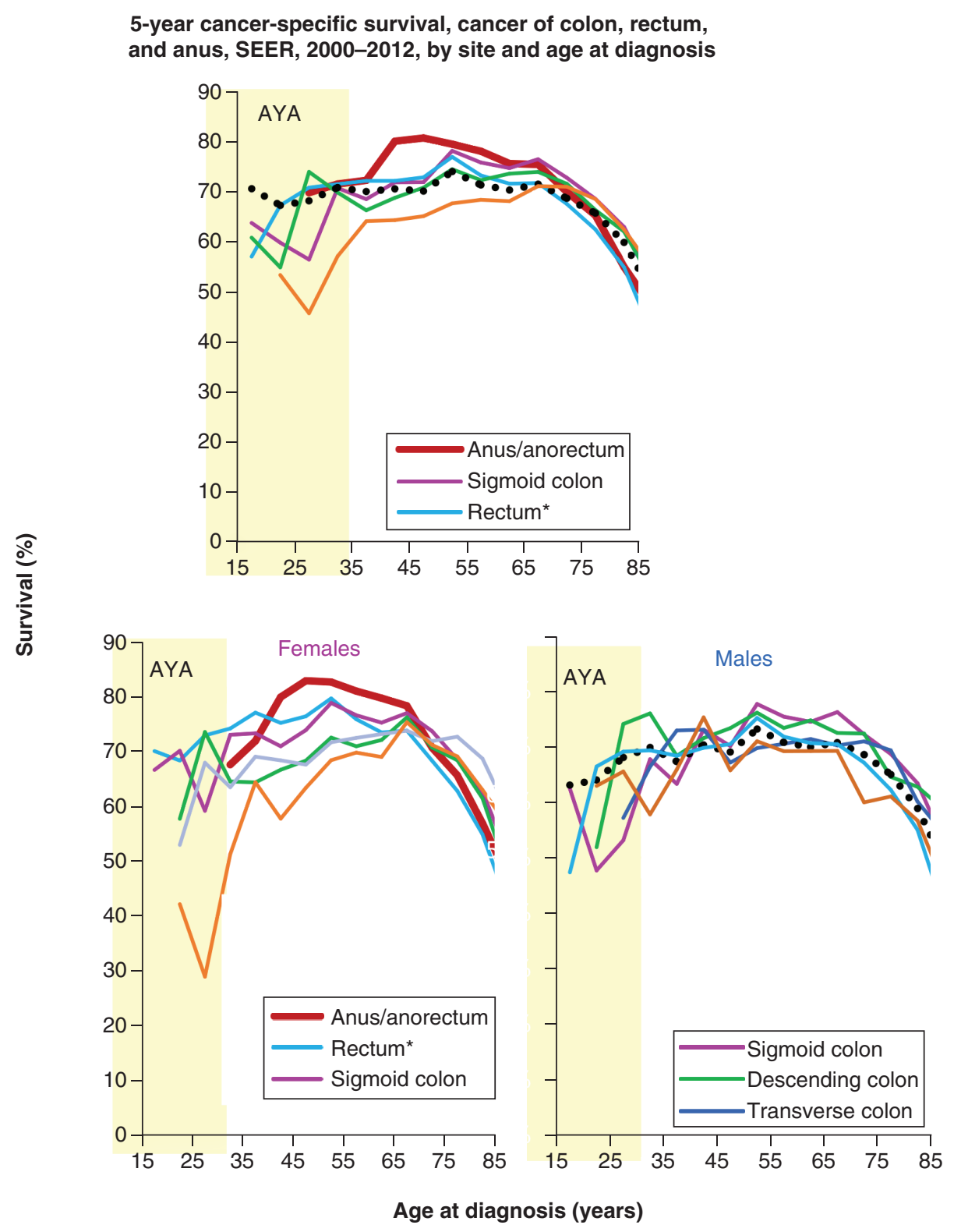

Figure 2. Incidence of invasive cancer of the colon, rectum and anus. Surveillance, epidemiology and end results 18 , 2000-2012 by age, site and sex. Age groups with $<10$ patients excluded (Tricoli et al. [35]).

*Includes rectosigmoid junction.

AYA: Adolescent and young adult.

increased frequency of $F B X W 7$ mutations in these patients, when compared with adults [40,41]. This indicates that findings are not always consistent across studies. Both AYACRC and EOCRC exhibit a lower frequency of KRAS mutations than those observed in CRC from older patients [42].

In a whole exome sequencing study comparing CRC tumors from AYA patients and adult patients, investigators detected 43 genes that met the criteria for exhibiting a significantly greater mutational frequency in AYA versus adult CRCs $(\mathrm{p}>0.05)$, based on gene function and/or evidence from the literature demonstrating the gene had a potential role in CRC [41]. Of these genes, 13 had a p-value $<0.01$ using the Fisher exact test and thus exhibited the greatest differential in mutation frequency between the AYA and adult groups. These include ROBO1, SIPAL1, MYCBP2, MED12L, BRCA2, MSH2, COL16A1, DNAJC2, EPHA3, LAMB2, MAP3K3, MCPH1 and RASGRP3. Most of the mutations detected in these genes were nonsynonymous missense, stop-gain or frameshift mutations that are damaging. Genes exhibiting alterations in mutational frequency in EOCRC and AYACRC are shown in 
Table 1. Genes with altered mutational frequency and altered miRNA expression in early-onset and adolescent and young adult colorectal cancer compared with adults.

\begin{tabular}{|c|c|c|c|c|c|}
\hline \multirow[t]{2}{*}{ Gene } & EOCRC mutation frequency & \multirow[t]{2}{*}{ Gene } & AYACRC mutation frequency & \multirow[t]{2}{*}{ miRNA } & EOCRC expression \\
\hline & Increase/decrease & & Increase/decrease & & Increase/decrease \\
\hline TP53 & Increase & ROBO1 & Increase & mir-106a & Increase \\
\hline CTNNB1 & Increase & SIPAL1 & Increase & mir-145 & Increase/decrease \\
\hline POLE & Increase & MYCBP2 & Increase & mir-125b & Decrease \\
\hline$A P C$ & Decrease & MED12L & Increase & $\operatorname{mir}-143$ & Decrease \\
\hline KRAS & Decrease & BRCA2 & Increase & $\operatorname{mir}-21$ & Increase \\
\hline$B R A F$ & Decrease & MSH2 & Increase & mir-20a & Increase \\
\hline PIKЗCA & Decrease & COL16A1 & Increase & $\operatorname{mir}-181 b$ & Increase \\
\hline FAM123B & Decrease & DNAJC2 & Increase & mir-203 & Increase \\
\hline \multirow[t]{8}{*}{ ATM } & Increase & EPHAЗ & Increase & $\operatorname{mir}-30 a-5 p$ & Decrease \\
\hline & & LAMB2 & Increase & mir-192 & Decrease \\
\hline & & МАРЗКЗ & Increase & $\operatorname{mir}-215$ & Decrease \\
\hline & & MCPH1 & Increase & mir-34a & Decrease \\
\hline & & RASGRP3 & Increase & mir-196a2 & Increase \\
\hline & & ATR & Increase & $\operatorname{mir}-27 a$ & Altered binding to target \\
\hline & & POLE & Increase & & \\
\hline & & & Increase & & \\
\hline \multicolumn{6}{|c|}{ EOCRC age range: $21-50$ years } \\
\hline \multicolumn{6}{|c|}{ AYACRC age range: $15-39$ years } \\
\hline $\begin{array}{l}\text { AYA: Adole } \\
\text { Data taken }\end{array}$ & oung adult; CRC: Colorectal can & CRC: Early & ectal cancer. & & \\
\hline
\end{tabular}

Table 2. Comparison of the differences in mutational frequency between the current adolescent and young adult colorectal cancer and adult colorectal cancer samples based on different levels of rigor.

\begin{tabular}{|c|c|c|c|}
\hline Gene & Exome data & Exome mutations validated by RNASeq & $\begin{array}{l}\text { Exome mutations validated by RNASeq } \\
\text { and are damaging }\end{array}$ \\
\hline MYCBP2 & AYA $50 \%$ Adult $7 \%$ & AYA $47 \%$ Adult $14 \%$ & AYA $41 \%$ Adult $0 \%$ \\
\hline BRCA2 & AYA $38 \%$ Adult $3 \%$ & AYA $47 \%$ Adult $14 \%$ & AYA $29 \%$ Adult $0 \%$ \\
\hline TOPORS & AYA $31 \%$ Adult $3 \%$ & AYA $29 \%$ Adult $0 \%$ & AYA $24 \%$ Adult $0 \%$ \\
\hline ATR & AYA $48 \%$ Adult $13 \%$ & AYA $41 \%$ Adult $7 \%$ & AYA $24 \%$ Adult $0 \%$ \\
\hline POLE & AYA $22 \%$ Adult $13 \%$ & AYA $24 \%$ Adult $14 \%$ & AYA $24 \%$ Adult $0 \%$ \\
\hline
\end{tabular}

Table 1. Further validation of the 43-gene set using RNASeq technologies revealed that five of these genes $M Y C B P 2$, (a MYC binding protein that possibly facilitates MYC) BRCA2, (involved in double strand DNA repair) TOPORS, (TOPO1 binding protein that regulates p53) ATR (PI3/PI4 kinase that phosphorylates BRCA1 and checkpoint proteins CHK1, RAD17 and RAD9) and POLE (catalytic subunit of DNA polymerase epsilon, DNA replication and repair) all demonstrated a statistically significant increase in the frequency of mutation in AYACRC compared with adult CRC, and displayed damaging mutations [Table 2]. It is very possible that these genes are also linked to EOCRC, certainly within the 21-39-year-old age group that overlaps with AYACRC patients and should be investigated further for their contribution to this disease.

Further validation of mutational gene variants associated with EOCRC and AYACRC will be required to narrow the population of gene variant sets and their associated pathways, which could be uniquely linked to CRC in younger patients. Eventually, focused in vivo and in vitro studies on specific genes will be required to discern the functional role of their products in EOCRC and AYACRC. Once the role of these genes is discerned, further investigations into the impact of alterations in these genes on disease development and progression and whether they provide opportunities to disrupt signaling pathways that can be targeted by precision medicine agents, can be conducted. 


\section{MIRNA \& EOCRC}

miRNAs are small (22-25 nucleotides in length) noncoding RNAs that can effectively alter the translation of target mRNAs by binding to their 3' untranslated region. A variety of studies have discovered the ability of individual miRNAs to regulate the expression of oncogenes and tumor suppressor genes, while others have demonstrated that miRNA gene loss or mutation can contribute to tumorigenesis. miRNA expression patterns (or signatures) are now known, in some cases, to characterize the developmental origins of tumors more effectively than messenger RNA expression signatures can sometimes provide. Evidence suggests that miRNAs may contribute to EOCRC and up to 200 miRNAs have been observed to be deregulated in CRC [43,44]. Thus, the potential for the disruption of normal signaling networks through mutation or deletion of miRNA is significant. In a Turkish cohort of EOCRC patients it was shown that expression of mir-106a was upregulated, while mir-145 and mir-125b were downregulated when compared with normal tissue [45]. While the fold changes in these miRNAs were modest, and no direct comparison was made with LOCRC, there was an association between the changes in these miRNAs and the presence of lymph node metastases in the patients studied. The collective gene targets for these miRNAs include many that are deregulated in CRC including BCL2, TP53, AKT1 BAX, CCND1, JUN, KRAS, MAPK3, MYC, ERK5, TNF, TGFBR2, CDKN1A, DICER1, E2F1, PTEN and RB1. Therefore, these miRNAs could be involved in the development or progression of EOCRC. For example, miR-143 can regulate cell growth and proliferation in vitro by targeting different oncogenic protein-coding genes, such as IRS-1, cMYC, MACC1, and KRAS [46-48].

Another study demonstrated significantly increased expression levels of mir-21, mir-20a, mir-145, mir 181b and mir-203 [49]. The increase in mir-145 expression contrasts with what the previous study reported; however, the investigators of this study point out that the observed increases in mir-145 expression may have been influenced by neoadjuvant therapy. Another miRNA, mir-30a-5p, while not known to specifically contribute to EOCRC, is a tumor suppressor miRNA that has been shown to target denticleless $(D T L)$ and was determined to be overexpressed in $95.8 \%$ of human CRC [50]. Two other miRNAs, mir-192 and mir-215, have been revealed to regulate $\mathrm{p} 53$ to suppress CRC [51], and another mir-34a has been shown to inhibit cellular invasion in CRC by targeting FRA1, a gene that encodes a encode leucine zipper protein that can dimerize with proteins of the JUN family, to form the AP-1 transcription factor complex [52]. Another area of investigation is single nucleotide polymorphisms (SNPs) detected in miRNAs that can affect their binding. In a study of a Chinese population, a SNP in mir-196a2 led to the increased expression of this miRNA and was associated with an increased CRC incidence in that group when compared with healthy controls [53]. Another study revealed that a SNP in mir-27a disrupts the binding of this miRNA to its target, the 3' untranslated region of $M B L 2$ and is associated with CRC risk in an African American population [54]. A summary of the miRNAs altered in EOCRC is presented in Table 1. Is it possible that similar miRNA SNPs are associated with EOCRC and AYACRC susceptibility? This is an avenue of investigation that should be vigorously pursued.

Many other miRNAs have been implicated in the biology of CRC [55], however, most have not been differentially examined for their involvement in EOCRC when compared with LOCRC. Identification of the miRNAs preferentially involved in EOCRC would provide new avenues into the understanding of the unique features of this disease and point the way toward useful biomarkers and more effective treatments for this disease.

\section{DNA repair pathway mutations}

DNA repair enzymes play an essential role in maintaining genomic integrity by correcting errors during DNA replication, and from environmental damage to the genome. Alterations in DNA repair pathways are a hallmark of EOCRC, particularly in the case of MSI-H disease, which is a feature of Lynch Syndrome [56]. In AYACRC tumors, one study reported that $39 \%$ contained at least one mutation in the DNA MMR gene MSH2, whereas only $3 \%$ of adult samples exhibited these mutations [41]. While this was expected because as in EOCRC, MSI is present in many AYA colon cancers, this study also reported a greater frequency of mutations in AYACRC patients for genes associated with other DNA repair pathways. These include BRCA2 (AYA samples, 39\%; adult samples, 3\%) and RAD9B (AYA samples, 22\%; adult samples, $0 \%$ ); and the cell-cycle checkpoint kinases $A T M$ (AYA samples, 35\%; adult samples, 7\%); ATR (AYA samples, 48\%; adult samples, $13 \%$ ) and POLE (AYA samples 24\%; adult samples 0\%) [Table 3]. POLE is a gene that encodes the catalytic subunit of DNA polymerase epsilon. The enzyme is involved in DNA repair and chromosomal DNA replication [16]. This gene was observed to be more frequently mutated in EOCRC compared with that identified in LOCRC patients [40]. This same gene was also noted to be more frequently mutated in AYACRC [41] and furthermore, several of the SNVs in the proofreading domain of POLE overlapped between the two studies. In one study, biallelic mutations in the MUTYH, a DNA 
Table 3. Genes associated with DNA repair pathways displaying significantly increased mutational frequency in adolescent and young adult colorectal cancer.

\begin{tabular}{|c|c|c|c|}
\hline Gene & Function & Mutation frequency in AYA (\%) & Mutation frequency in adult (\%) \\
\hline MSH2 & Mismatch repair & 39 & 3 \\
\hline MCPH1 & DNA damage response & 26 & 0 \\
\hline$R A D 9 B$ & DNA repair endonuclease & 22 & 0 \\
\hline ATM & Cell-cycle checkpoint kinase & 35 & 7 \\
\hline ATR & $\mathrm{PI} 3 / \mathrm{PI} 4$ kinase & 48 & 13 \\
\hline POLE & DNA repair & 24 & 0 \\
\hline
\end{tabular}

glycosylase involved in oxidative DNA damage repair, accounted for 3-4\% of early-onset CRC without apparent polyposis in MMR-proficient patients [57]. Base excision repair deficiency, due to biallelic inactivation of MUTYH, has previously been associated with the development of an attenuated form of colonic polyposis [58]. Mutations in DNA repair pathway genes may provide therapeutic targets, as has been the case with PARP (poly ADP ribose polymerase) inhibitors for the treatment of tumors with mutations in homologous recombinational repair, such as BRCA1, BRCA2 and PALB2 [59]. Therefore, sporadic mutations in DNA-repair genes, other than the MMR genes (MLH1, MSH2 and MSH6), may be important to the biology and development of colon cancer in these patient populations.

A survey of comparing EOCRC and LOCRC using targeted DNA repair panels and NGS assays could be very beneficial in elucidating the role of repair pathways, beyond those associated with MSI, in the EOCRC patients.

\section{Future perspective}

In order to 'move the field' for both EOCRC and AYACRC, we must expand and extend the previous and current work on the basic biology and translational research related to this disease. As we have seen from the data summarized above, findings across studies are not always consistent. Therefore, future studies need to be cross validated utilizing different sequencing platforms to verify interesting variants. The identification of novel genes and pathways associated with EOCRC should not be limited to the use of gene panels but will require the use of whole exome sequencing in order to survey the entire coding region of the genome. The analysis of miRNA profiles, their target genes and polymorphisms that impact their binding to those genes will also be important in understanding the biology of EOCRC and could point the way to novel biomarkers for early disease detection. DNA repair pathways should be a particular focus of future studies since these alterations appear to be very consistent among EO- and AYA CRC patients. One example is POLE being identified as more frequently mutated in two studies using disparate patient populations and different sequencing platforms and calling algorithms. Thus, repair pathways outside of those associated with hereditary nonpolyposis colorectal cancer, FAP and MSI should be extensively investigated for mutations that can impact disease development. Once these genes/pathways are identified they must then be validated on larger sample sets which will require surveying available biobanks for these patient samples. Identification of EOCRC and AYACRC samples can be challenging, particularly in the younger cohorts, due to the rarity of these cancers. Efforts must be made to better catalog and identify these samples, along with annotated clinical information and pre-analytics, within existing biobanks and make them available for analysis. Ultimately, identifying novel genes/pathways associated with EOCRC will provide the molecular foundations for the development of novel precision medicine treatments for this disease and lead to improved survival for this cohort of patients.

\section{Acknowledgments}

The author would like to thank $\operatorname{Dr}$ A Bleyer for the contribution of Figure 1 and for valuable consultation on its interpretation.

Financial \& competing interests disclosure

The author has no relevant affiliations or financial involvement with any organization or entity with a financial interest in or financial conflict with the subject matter or materials discussed in the manuscript. This includes employment, consultancies, honoraria, stock ownership or options, expert testimony, grants or patents received or pending, or royalties.

No writing assistance was utilized in the production of this manuscript. 
Open access

This work is licensed under the Attribution-NonCommercial-NoDerivatives 4.0 Unported License. To view a copy of this license, visit http://creativecommons.org/licenses/by-nc-nd/4.0/

\section{Executive summary}

- Differences in mutational profiles for early-onset colorectal cancer (EOCRC) and adolescent and young adult colorectal cancer (CRC), compared with adult colorectal cancer have been identified that will require validation and follow-up.

- Additional studies are required using more expansive next-generation sequencing platforms, such as whole-exome sequencing, to identify novel genes that may exhibit a greater mutational frequency in EOCRC and adolescent and young adult CRC compared with late-onset CRC and could contribute to this disease.

- Results from mutational profile comparisons between EOCRC and late-onset CRC should be pursued using in vivo and in vitro studies to better elucidate the role of these genes in the biology of this disease.

- Identification of novel mutational profiles in this disease should be utilized to identify biomarkers that can be used for early disease detection.

- miRNAs in EOCRC should be further investigated to elucidate the possible unique biology of this disease and identify therapeutic targets.

- Alterations in DNA repair pathway genes should be further characterized in this disease for the purpose of identifying therapeutic targets.

\section{References}

Papers of special note have been highlighted as: $\bullet$ of interest

1. Seigel RL, Fedewa SA, Anderson WF et al. Colorectal cancer incidence patterns in the United States, 1974-2013. J. Natl Cancer Inst. 109(8), doi: 10.1093/jnci/djw322 (2017) (Epub ahead of print).

2. Seigel RL, Medhanie GA, Fedewa SA et al. State variation in early onset colorectal cancer in the United States, 1995-2015. J. Natl Cancer Inst. 111(10), 1104-1106 (2019).

3. Ganz PA. Current US cancer statistics: alarming trends in young adults? J. Natl Cancer Inst. 111(12), 1241-1242 (2019).

4. Vuik F, Nieuwenburg S, Bardou M et al. Increasing incidence of colorectal cancer in young adults in Europe over the last 25 years. Gut 68(10), 1820-1826 (2019).

5. Tricoli JV, Blair DG, Anders CK et al. Biologic and clinical characteristics of adolescent and young adult cancers: acute lymphocytic leukemia, colorectal cancer, breast cancer, melanoma and sarcoma. Cancer 122(7), 1017-1028 (2016).

6. Tricoli JV, Bleyer A. Adolescent and young adult cancer biology. Cancer J. 24(6), 267-274 (2018).

7. Bleyer A, Barr R, Hayes-Lattin B et al. The distinctive biology of cancer in adolescents and young adults. Nat. Rev. Cancer 8(4), 288-298 (2008).

- This was one of the earliest comprehensive publications on the distinctive biology of adolescent and young adult cancers.

8. Hubbard JM, Grothey A. Adolescent and young adult colorectal cancer. J. Natl Comp. Cancer Netw. 11(10), 1219-1225 (2013).

9. Liang JT, Huang KC, Cheng AL et al. Clinicopathological and molecular biological features of colorectal cancer in patients less than 40 years of age. Br. J. Surgery 90(2), 205-214 (2003).

10. Ballester WJ, Shahrooz R, Boardman L. Clinical and molecular features of young-onset colorectal cancer. World J. Gastroenterol. 22(5), 1736-1744 (2016).

11. Berg M, Agesen TH, Thiis-Evensen E et al. Distinct high-resolution genome profiles of early onset and late onset colorectal cancer integrated with gene expression data identify candidate susceptibility loci. Mol. Cancer 9(100), doi: 10.1186/1476-4598-9-100 (2010) (Epub ahead of print).

12. Hur K, Cejas P, Feliu J et al. Hypomethylation of long interspersed nuclear element-1 (LINE-1) leads to activation of proto-oncogenes in human colorectal cancer metastasis. Gut 63(4), 635-646 (2014).

13. Chen RZ, Pettersson U, Beard C et al. DNA hypomethylation leads to elevated mutation rates. Nature 395(6697), 89-93 (1998).

14. Holm TM, Jackson-Grusby L, Brambrink T et al. Global loss of imprinting leads to widespread tumorigenesis in adult mice. Cancer Cell $8(4), 275-285$ (2005).

15. de la Chapelle A. Genetic predisposition to colorectal cancer. Cancer 4(10), 769-780 (2004).

16. Weizmann Institute of Science. Gene Cards. Weizmann Institute of Science, Rehovot, Israel (2016). http://www.genecards.org/

17. Cummins JM, He Y, Leary RJ et al. The colorectal microRNAome. Proc. Natl Acad. Sci. 103(10), 3687-3692 (2006).

18. Giraldez MA, Lopez-Doriga A, Bujanda L et al. Susceptibility genetic variants associated with earl-onset colorectal cancer. Carcinogenesis 33(3), 613-619 (2012). 
19. Von Holst S, Picelli S, Elder D et al. Association studies on 11 published colorectal cancer risk loci. Br. J. Cancer 103(4), 575-580 (2010).

20. He J, Wilkens LJ, Stram DO et al. Generalizability and epidemiologic characterization of eleven colorectal cancer GWAS hits in multiple populations. Cancer Epidemiol. Biomarkers Prev. 20(1), 70-81 (2011).

21. Archambault AN, Su Y-R, Jeon J et al. Cumulative burden of colorectal cancer-associated genetic variants is more strongly associated with early-onset vs late-onset cancer. Gasteroenterology DOI: https://doi.org/10.1053/j.gastro.2019.12.012

22. Jung SM, Yoon YS, Lim SB et al. Clinicopathological features of familial adenomatous polyposis in Korean patients. World J. Gastroenterol. 22(17), 4380-4388 (2016).

23. Dalpatadu KU, Anwar N, Wijesuriya SR et al. Uses of a familial adenomatous polyposis registry. Ceylon Med. J. 56(2), 66-69 (2011).

24. Jiang W, Cai MY, Li SY et al. Universal screening for Lynch syndrome in a large consecutive cohort of Chinese colorectal cancer patients: high prevalence and unique molecular features. Int. J. Cancer 144(9), 2161-2168 (2019).

25. Arriba M, Sánchez C, Vivas A et al. Intermediate-onset colorectal cancer: a clinical and familial boundary between both early and late-onset colorectal cancer. PLoS ONE 14(5), e0216472 (2019).

26. Sjoblom T, Jines S, Wood LD et al. The consensus coding sequence of human breast and colorectal cancers. Science 314(5797), 268-274 (2006).

27. Wood LD, Parsons DW, Jones S et al. The genomic landscapes of human breast and colorectal cancers. Science 318(5853), 1108-1113 (2007).

28. Esteban-Jurado C, Gimenez-Zaragoza D, Munoz J et al. POLE and POLD1 screening in 155 patients with multiple polyps and early-onset colorectal cancer. Oncotarget 8(16), 26732-26743 (2017).

29. Lieu CH, Golemis EA, Serebriiskii IG et al. Comprehensive genomic landscapes in early and later onset colorectal cancers. Clin. Cancer Res. 25(19), 5852-5858 (2019).

30. Dwyer AJ, Murphy CC, Boland R et al. A summary of the fight colorectal cancer working meeting: exploring risk factors and etiology of sporadic early-age onset colorectal cancer. Gastroenterology 157(2), 280-288 (2019).

31. Willauer AN, Liu Y, Pereira AL et al. Clinical and molecular characterization of early-onset colorectal cancer. Cancer 125(12), 2002-2010 (2019).

32. Bonilla C, Lefevre JH, Winney B et al. Cyclin D1 rare variants in UK multiple adenoma and early-onset colorectal cancer patients. J. Human Genet. 56(1), 58-63 (2011).

33. Kirzin S, Marisa L, Guimbaud R et al. Sporadic early-onset colorectal cancer is a specific sub-type of cancer: a morphological, molecular, and genetics study. PLoS ONE 9(8), 1-13 (2014).

34. Agesen TH, Berg M, Clancy T et al. CLC and INFAR1 are differentially expressed and a global immunity score is distinct between earlyand late-onset colorectal cancer. Genes Immun. 12(8), 653-662 (2011).

35. Tricoli JV, Anninga J. The biology of AYA cancers. In: Cancers in Adolescents and Young Adults (2nd Edition). Ries L, Whelan J, Ferrari A, Bleyer A, Barr R. (Eds). Springer International Publishing, Cham, Switzerland, $43-67$ (2017).

36. Liu B, Farrington SM, Petersen GM et al. Genetic instability occurs in the majority of young patients with colorectal cancer. Nat. Med. 1(4), 348-352 (1995).

37. Kakar S, Saime A, Burgart LJ et al. Mucinous carcinoma of the colon: correlation of loss of mismatch repair enzymes with clinicopathologic features and survival. Mod. Pathol. 17(6), 696-700 (2004).

38. Durno C, Aronson M, Bapat B et al. Family history and molecular features of children, adolescents, and young adults with colorectal carcinoma. Gut 54(8), 1146-1150 (2005).

39. Hong Y, Ho KS, Eu KW et al. A susceptibility gene set for early onset colorectal cancer that integrates diverse signaling pathways: implication for tumorigenesis. Clin. Cancer Res. 13(4), 1107-1114 (2007).

40. Kothari N, Teer JK, Abbott AM et al. Increased incidence of FBXW7 and POLE proofreading domain mutations in young adult colorectal cancers. Cancer 122(18), 2828-2835 (2016).

- This was one of the first comprehensive genomic analysis studies on young adult colon cancer.

41. Tricoli JV, Boardman LA, Patidar R et al. A mutational comparison of adult and adolescent and young adult (AYA) colon cancer. Cancer 124(5), 1070-1082 (2018).

- This was the first study to use whole-exome sequencing to identify differences in mutational variants between adolescent and young adult and adult colon cancers.

42. Chang DT, Pai RK, Rybicki LA et al. Clinicopathologic and molecular features of sporadic early-onset colorectal adenocarcinoma: an adenocarcinoma with frequent signet ring cell differentiation, rectal and sigmoid involvement, and adverse morphologic features. Mod. Pathol. 25(8), 1128-1139 (2012).

43. Volinia S, Calin GA, Liu CG et al. A microRNA expression signature of human solid tumors defines cancer gene targets. Proc. Natl Acad. Sci. 103(7), 2257-2261 (2006).

44. Nugent M, Miller N, Kerin MJ. Micro RNAs in colorectal cancer: function, dysregulation and potential as novel biomarkers. Eur. J. Surg. Oncol. 37(8), 649-654 (2011). 
45. Ak S, Tunca B, Tezcan G et al. MicroRNA expression patterns of tumors in early-onset colorectal cancer. J. Surg. Res. 191(1), 113-122 (2014).

46. Schetter AJ, Okayama H, Harris CC. The role of microRNAs in colorectal cancer. Cancer J. 18(3), 244-252 (2012).

47. Michael MZ, O Conner SM, Pellekaan vH et al. Reduced accumulation of specific microRNAs in colorectal neoplasia. Mol. Cancer Res. 1(12), 882-891 (2003).

48. Zhang Y, Wong Z, Chen M et al. MicroRNA-143 targets MACC1 to inhibit invasion and migration in colorectal cancer. Mol. Cancer $\mathrm{X}(11), 00-00$ (2012).

49. Yantiss RK, Goodarzi M, Zhou XK et al. Clinical, pathologic, and molecular features of early-onset colorectal carcinoma. Am. J. Surg. Path. 33(4), 572-582 (2009).

50. Baraniskin A, Birkenkamp-Demtroder K, Maghnouj A et al. MiR-30a-5p suppresses tumor growth in colon carcinoma by targeting DTL. Carcinogenesis 33(4), 732-739 (2012).

51. Braun CJ, Zhang X, Savelyeva I et al. p-53 responsive microRNAs 192 and 215 are capable of inducing cell cycle arrest. Cancer Res. 68(24), 10094-10104 (2008).

52. Wu J, Wu G, Lv L et al. MicroRNA-34a inhibits migration and invasion of colon cancer cells via targeting to Fra-1. Carcinogenesis 33(3), 519-528 (2012).

53. Zhan JF, Chen LH, Chen ZX et al. A functional variant in microRNA $196 \mathrm{a} 2$ is associated with susceptibility of colorectal cancer in a Chinese population. Arch. Med. Res. 42(2), 144-148 (2011).

54. Zanetti KA, Haznadar M, Welsh JA et al. 3'-UTR and functional secretor haplotypes in mannose-binding lectin 2 are associated with increased colon cancer risk in African Americans. Cancer Res. 72(6), 1467-1477 (2012).

55. Chen B, Xia Z, Deng Y-N et al. Emerging microRNA biomarkers for colorectal cancer diagnosis and prognosis. Open Biology 9(1), 180212 (2019).

56. Lynch JT, Lynch JF, Lynch PM et al. Hereditary colorectal cancer syndromes: molecular genetics, genetic counseling, diagnosis and management. Fam. Cancer 7(1), 27-39 (2008).

57. Giraldez MA, Balaguer F, Bujanda L et al. MSH6 and MUTYH deficiency is a frequent event in early-onset colorectal cancer. Clin. Cancer Res. 16(22), 5402-5413 (2010).

58. Al Tassen N, Chmiel NH, Maynard J et al. Inherited variants of MYH associated with somatic G:C-> T:A mutations in colorectal tumors. Nat. Genetics 30(2), 227-232 (2002).

59. Buisson R, Dion-Cote AM, Coulombe $\mathrm{Y}$ et al. Cooperation of breast cancer proteins PALB2 and piccolo BRCA2 in stimulating homologous recombination. Nat. Struct. Mol. Biol. 17(10), 1247-1254 (2010). 